\title{
Newton black films as wetting systems
}

\author{
Pedro Tarazona, ${ }^{1,2, *}$ Hector Martínez, ${ }^{1, \dagger}$ Enrique Chacón, ${ }^{2,3, \dagger}$ and Fernando Bresme ${ }^{4, \S}$ \\ ${ }^{1}$ Departamento de Física Teórica de la Materia Condensada, Universidad Autónoma de Madrid, Madrid, ES-28049, Spain \\ ${ }^{2}$ Instituto de Ciencia de Materiales Nicolás Cabrera, Universidad Autónoma de Madrid, Madrid, ES-28049, Spain \\ ${ }^{3}$ Instituto de Ciencia de Materials de Madrid, CSIC, Madrid, ES-28049, Spain \\ ${ }^{4}$ Department of Chemistry, Imperial College London, SW7 2AZ, London United Kingdom
}

(Received 12 January 2012; published 2 February 2012)

\begin{abstract}
Newton black films (NBFs) can appear under a wide range of experimental conditions. NBFs define the adhesive states of foams and emulsions, showing their formation is a very general physical phenomenon. We show that the existence of NBFs and their whole experimental behavior can be understood within the theory of wetting transitions. NBFs are experimental realizations of partial wetting or pre-wetting states. Hence, they provide experimental systems to investigate the pre-wetting transition, and the spreading behavior under conditions that are very difficult to realize in other experimental systems. We also introduce two new computational approaches to obtain the disjoining pressure isotherm from canonical simulations, and to estimate the contact angles of droplets of nanoscopic dimensions.
\end{abstract}

DOI: 10.1103/PhysRevB.85.085402

PACS number(s): 68.15.+e, 05.70.Np, 68.35.Md, 68.35.Rh

\section{INTRODUCTION}

The iridescent films often observed in soap bubbles can lose water and transform into a thinner black film, which does not reflect light. Newton ${ }^{1}$ appears to be the first reporting the existence of these so called Newton black films (NBFs). NBFs are self-assembled structures consisting of nanometer thick layers of water stabilized by surfactants. ${ }^{2}$ They can be stabilized with a wide variety of amphiphilic molecules, ionic, nonionic as well as phospholipids. ${ }^{3,4}$ Two distinct types of films can be observed depending on the experimental conditions. Common black films (CBFs) have a thickness $h>5 \mathrm{~nm}$, which varies with the ionic strength of the solution as predicted by the Poisson-Boltzmann theory (PBT). ${ }^{5,6}$ For smaller thicknesses experimental and theoretical studies have reported significant deviations from the PBT. ${ }^{7,8}$ At high salt concentration and low temperatures, CBFs undergo a transition to a thinner Newton black film (NBF) that is stable in the absence of external disturbances, and its thickness $(h \approx 3-4 \mathrm{~nm})$ is almost insensitive to the amount of salt, temperature, or pressure. It has been argued that NBFs are always metastable with respect to their rupture, ${ }^{9}$ but the high metastability barrier would allow treating them as thermodynamic equilibrium states, under the constraint of constant film area. ${ }^{10}$ The disjoining pressure $\Pi$, defined as the pressure difference between the vapor outside and the water inside the film, is often used to stabilize NBFs using the thin-film balance technique. ${ }^{3,7,11} \Pi$ controls (see Section III) the shift of the chemical potential of water, $\Delta \mu=\mu_{\text {sat }}-\mu$, with respect to its liquid-vapor saturation value. NBFs can form spontaneously at $\Pi=0$, or upon application of an external pressure, $\Pi>0 .{ }^{11}$ Several experiments have highlighted the existence of hysteresis in the CBF and NBF transition, ${ }^{7,11}$ showing the transition might be first order. The continuous thinning of the film under increasing $\Pi$ has also been reported. ${ }^{11}$

Microscopically, the interactions determining the formation of NBF are similar in nature and strength to those regulating the stability of colloidal suspensions and emulsions. Emulsion droplets feature strong attractions that lead to adhesion, and the droplets stick together avoiding coalescence. ${ }^{12}$ The resulting interdroplet film is a NBF, and its structure is remarkably similar to NBFs formed in foams. ${ }^{13,14}$ Adhesive droplets, ${ }^{15}$ including biological vesicles, ${ }^{16}$ feature well defined contact angles. Similarly, foam NBFs feature contact angles with thicker films. ${ }^{17}$ The analysis of these results indicates that the contact angles observed in emulsions and foams have the same physical origin.

In our view, these experimental observations are representative of a wide range of phenomena that can be described with the wetting transition (WT) theory. ${ }^{18-20}$ The water confined inside the film is the wetting phase, while the inner surface between the amphiphilic layers plays the role of the substrate in the usual WT systems. To establish the connection between NBF formation and wetting, we focus on the geometry of the water droplets inside the film, and on the role of the disjoining pressure to control the undersaturation of water with respect to its bulk liquid-vapor coexistence. The interaction between the amphiphile and water and the salinity of water define the wetting temperature and the prewetting branch in the WT theory. Newton in his experiments ${ }^{1}$ of NBF exercised great care to avoid external disturbances and the evaporation of water by covering the films in a "clear glass." This experimental setup would achieve two objectives: (1) avoid that the system crosses the metastability barrier for rupture and (2) keep the water vapor at coexistence with the liquid, i.e., $\Delta \mu=0$ or $\mu_{\text {sat }}=\mu$. The (under)saturation of the wetting phase is precisely the key control parameter in the phenomenology of wetting, and the interpretation of the phenomenology of NBF and (CBF as a particular case of a wetting system.

In this paper, we show that the formation of NBF is representative of a more general phenomenology that can described using the WT theory. Establishing this connection, enable us to identify the formation of black films as either partial wetting or prewetting transitions.

Our paper is structured as follows. Firstly, we briefly discuss the computer simulation methodology employed in this work. We then discuss the two main approaches to quantify the wetting transition in the films, namely, the control of the undersaturation of the water vapor in experiments of soap 
films, and the geometry of the water droplets inside the film. To address the first point, we present an approach to quantify the change in the chemical potential, and hence, the disjoining pressure, from canonical simulations. We then present a comprehensive study of droplet formation inside NBFs. A discussion of the impact of the size of the simulation box on the geometry of the droplets follow, and we introduce a novel approach to compute the contact angle of the resulting nanoscopic droplets. The formation of NBF in experiments and computer simulations are then discussed in terms of the WT theory. This is the main contribution from this work. A final section with the main conclusions and final remarks closes the paper.

\section{COMPUTER SIMULATIONS}

The computer simulations were performed at constant temperature, $T=298$ and $375 \mathrm{~K}$ in the canonical ensemble $(\mathrm{N}, \mathrm{V}, \mathrm{T})$ using the Nosé-Hoover thermostat with a coupling constant of $0.5 \mathrm{ps}$. The simulations were performed in parallel using the code GROMACS $4.5 .^{21}$

We performed simulations with films containing different number of surfactants, $N_{\text {SDS }}=512$ and 2048 sodium dodecyl sulfate (SDS) surfactants, with $N_{\text {SDS }} / 2$ surfactants per monolayer. $N_{\text {SDS }}$ sodium counterions were randomly added, and the number of water molecules, $N_{w}$, was varied between $N_{w} / N_{\text {SDS }}=1$ and 12 , to achieve different film thicknesses. The largest systems (2048 SDS) were employed to simulate adhesive films and to compute the contact angles.

The surfactants were modeled using united atom force fields and the water molecules using the TIP4P-2005 rigid model. ${ }^{22}$ Full details of the force-field parameters can be found in Refs. 23 and 24. The van der Waals interactions were truncated using a spherical cutoff of $14 \AA$. The electrostatic interactions were computed using the particle mesh Ewald method. ${ }^{25}$

The simulations were performed over $200 \mathrm{~ns}$ at $298 \mathrm{~K}$ and $50 \mathrm{~ns}$ at $375 \mathrm{~K}$ with 100 and $25 \mathrm{~ns}$ of equilibration, respectively, and the equations of motion were integrated using a time step of 0.002 ps. The area per surfactant in our simulations was set to $33 \AA^{2}$, corresponding to the experimental estimate in SDS NBFs. ${ }^{13}$

All the films were initially positioned on the $x y$ plane of the simulation box with large vacuum regions above and below the film, rendering a simulation box that is fully periodic. Upon adhesion, a NBF in coexistence with a water droplet was formed, but only after the long equilibration times reported above and using large system sizes, $N_{\mathrm{SDS}}=2048$ (see Ref. 24).

The surface tensions were computed through the microscopic pressure tensor,

$$
\gamma=L_{z}\left[P_{z z}-\frac{1}{2}\left(P_{x x}+P_{y y}\right)\right]
$$

where $P_{\alpha \beta}$ are the pressure tensor components with $\alpha$ and $\beta$ equal to the cartesian coordinates, $(x, y, z)$, and $L_{z}$ the box length in the direction normal to the interface plane. The pressure components were obtained from the virial equation,

$$
P_{\alpha \beta} V=\sum_{i=1}^{N} m_{i} \mathbf{v}_{i, \alpha} \mathbf{v}_{i, \beta}+\sum_{i=1}^{N-1} \sum_{j>i}^{N} \mathbf{r}_{i j, \alpha} \mathbf{f}_{i j, \beta},
$$

where $\mathbf{v}_{i, \alpha}$ represents the velocity of atom $i$ in direction $\alpha, \mathbf{r}_{i j}$ is the vector joining atoms $i$ and $j$, and $\mathbf{f}_{i j}$ is the force between these two atoms.

\section{CONTROL OF THE UNDERSATURATION OF THE WATER VAPOR IN EXPERIMENTS AND SIMULATIONS OF SOAP FILMS}

In the following, we give a detailed account of the equivalence between the parameter $\Delta \mu$, which is the usual control parameter in WT theory and the disjoining pressure $\Pi$ used in experimental studies of CBF-NBF transitions. ${ }^{11}$ Also we show how to obtain $\Delta \mu$ from the results of canonical ensemble simulations using the Gibbs-Duhem relation.

The disjoining pressure is controlled by the difference between the vapor pressure outside the soap film $p_{\text {vap }}$ and the pressure of the liquid water reservoir connected to the soap film $p_{\text {liq }}$. The difference $\Delta p=p_{\text {vap }}-p_{\text {liq }}$ is balanced by the repulsion between the two monolayers so that $\Pi=\Delta p$. On the other hand, the chemical potential of water, $\mu$, has to be the same in the vapor outside the film and inside the soap bilayer. In absence of disjoining pressure $\Pi=0, p\left(\rho_{\text {liq }}\right)=p\left(\rho_{\text {vap }}\right)=$ $p_{\text {coex }}$, and $\mu\left(\rho_{\text {liq }}\right)=\mu\left(\rho_{\text {vap }}\right)=\mu_{\text {coex }}$. These conditions define the densities of the coexisting liquid, $\rho_{\text {liq }}$, and vapor, $\rho_{\text {vap }}$, phases at each temperature. The pressure in the vapor phase can be easily changed in the experiments. The shifts in the densities of the liquid and vapor from the coexisting values at $\Pi=0$ are given by the solution of the coupled equations:

$$
\begin{aligned}
& p\left[\rho_{\text {liq }}+\Delta \rho_{\text {liq }}(\Pi)\right]=p\left[\rho_{\text {vap }}+\Delta \rho_{\text {vap }}(\Pi)\right]+\Pi, \\
& \mu\left[\rho_{\text {liq }}+\Delta \rho_{\text {liq }}(\Pi)\right]=\mu\left[\rho_{\text {vap }}+\Delta \rho_{\text {vap }}(\Pi)\right]+\Delta \mu(\Pi) .
\end{aligned}
$$

Considering the Gibbs-Duhem relation for the bulk phases, at constant temperature, we get

$$
\begin{gathered}
p\left[\rho_{\mathrm{liq}}+\Delta \rho_{\mathrm{liq}}(\Pi)\right]=p_{\mathrm{coex}}+\rho_{\mathrm{liq}} \Delta \mu(\Pi), \\
p\left[\rho_{\mathrm{vap}}+\Delta \rho_{\mathrm{vap}}(\Pi)\right]=p_{\mathrm{coex}}+\rho_{\mathrm{vap}} \Delta \mu(\Pi),
\end{gathered}
$$

and finally,

$$
\Delta \mu(\Pi)=\frac{\Pi}{\rho_{\text {liq }}-\rho_{\text {vap }}},
$$

which defines the shift in chemical potential of water inside the film with respect to the bulk coexisting value of water in the liquid reservoir.

The molecular dynamics (MD) simulation results for the surface tension $\gamma\left(N_{w}\right)$ as a function of the water content $N_{w}$ in a system with fixed area $A$ and number of SDS surfactants $N_{\text {SDS }}$ may be used to calculate the variation of the chemical potential of water and the equivalent disjoining pressure in an experimental setup. Since the volume of the vapor phase is small, the simulations results may be interpreted in terms of a surface Gibbs-Duhem relation $d \gamma=-\left(N_{w} / A\right) d \mu$. The results for the large system in which the water drop coexists with the equilibrium NBF give the value $N_{w}=N_{\mathrm{NBF}}$ at which $\mu\left(N_{w}\right)=\mu_{\text {coex }}$. Integrating we get

$$
\Delta \mu \equiv \mu_{\mathrm{coex}}-\mu\left(N_{w}\right)=\int_{N_{w}}^{N_{\mathrm{NBF}}} \frac{A}{N_{w}} \frac{d \gamma}{d N_{w}} d N_{w},
$$



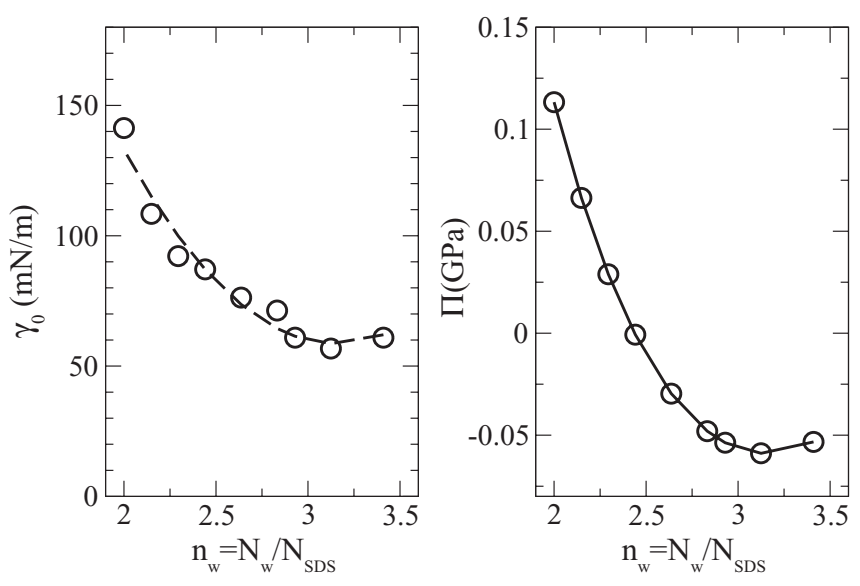

FIG. 1. (Left) Dependence of the film surface tension with water content. The dashed line represents a quadratic fit to the simulation data. (Right) The disjoining pressure isotherm obtained from the surface tensions and Eqs. (6) and (7). The solid line is a guide to the eye.

and the equivalent disjoining pressure

$$
\Pi\left(N_{w}\right)=\Delta \mu(\Pi)\left(\rho_{\text {liq }}-\rho_{\text {vap }}\right) .
$$

Figure 1 shows the results for surface tension obtained in the canonical ensemble MD simulations, using 256 SDS surfactants per monolayer, and the fit of the data to a quadratic equation. We consider in the fit surface tensions from $N_{w}$ well below the equilibrium $N_{\mathrm{NBF}}$ value, to the spinodal point, where $\gamma\left(N_{w}\right)$ reaches a minimum and, consequently, $\mu\left(N_{w}\right)$ is a maximum. The fit was used to obtain the disjoining pressure represented in the right panel of Fig. 1 using Eqs. (6) and (7) given above. Notice that the calculation cannot be extended beyond the minimum of $\gamma\left(N_{w}\right)$, since the homogeneous films become unstable, and they nucleate droplets that break the homogeneity assumed in the Gibbs-Duhem relation. This tendency to nucleate droplets is reduced for smaller simulation boxes, which show a larger metastable branch, and allow to explore homogeneous systems with $N_{w} / A$ ratios that would result otherwise in droplet nucleation.

\section{GEOMETRICAL PHASE DIAGRAM OF DROPS IN NEWTON BLACK FILMS}

In our simulations of SDS films with different amounts of water, we observe three qualitatively different drop structures. At low water content, the structure is a thin film of undersaturated NBF. For a water/surfactant ratio $n_{w} \approx 2$, the chemical potential of water reaches its saturation value, and any excess amount of water results in the formation of liquid droplets inside the SDS bilayer. However, in simulations with small cross sectional areas [e.g., $A=(9.2 \mathrm{~nm})^{2}$ ], the structure of the film remains globally uniform, as a metastable supersaturated NBF, up to a spinodal point approximately at $n_{w} \approx 3$. Increasing $n_{w}$ beyond that value, results in droplet formation, and the in-plane homogeneity of the film is broken. Following a slow process, the droplets coalesce into a single large drop, provided the cross sectional area, $A$ of the box is large enough. Further increase of the amount of water, $n_{w}>6$, leads to the formation of thick films that are again globally uniform as a result of the finite size of the simulation box and the periodic boundary conditions, which result in coalescence of the periodic images of the large water drops into a water slab, which now takes full advantage of the boundary conditions to accommodate any amount of water without increasing its surface area. This later effect may be understood in terms of a simple geometrical model in which we compare the surface excess free energy of a cap-shaped drop, which makes no use of the periodic boundary conditions on the $x y$ plane, with a cylindrical drop, which uses the periodic boundaries in the $x$ direction, and a planar slab, which uses the periodicity in both transverse directions. In each case, the system has a surface $A_{s}$ of liquid water covered by a SDS amphiphile and a surface $A_{f}$ of thin SDS film. For each geometry, the values of $A_{s}$ and $A_{f}$ have to be calculated with the constraints that the liquid water volume is constant, and the total transverse area $A$ is also constant. Hence, the excess free energy is calculated as $\Delta F=\gamma A_{s}+\gamma_{f} A_{f}$, with the surface tensions of the film $\gamma_{f}$ and that of the liquid water with the SDS monolayer $\gamma$. In our case, $\gamma_{f}$ corresponds to the result computed for the saturated $\mathrm{NBF}, \gamma_{f}=\gamma_{\mathrm{NBF}}=77 \mathrm{mN} / \mathrm{m}$ at $T=298 \mathrm{~K}$, and $\gamma$ is one half of the value computed for the thick slabs, $\gamma_{\text {thick }}=120 \mathrm{mN} / \mathrm{m}$, which corresponds to $A_{f}=0, A_{s}=$ $2 A$, and hence $\Delta F_{\text {slab }}=\gamma_{\text {thick }} A$. The optimal shapes for the spherical caps or the cylindrical segment have to be obtained with the contact angle $\theta$ that fulfills the mechanical equilibrium condition set by Young's equation $\cos \theta=\gamma_{f} /(2 \gamma)=$ $\gamma_{\mathrm{NBF}} / \gamma_{\text {thick }}$. Therefore the volume of the spherical double cap drop, $V=4 \pi / 3(1-\cos \theta)^{2} R^{3}$, determines its radius $R$, the areas $A_{s}=4 \pi(1-\cos \theta) R^{2}$, and $A_{f}=A-\pi(\sin \theta)^{2} R^{2}$, and hence its free energy $F_{\text {sph }}$. Similarly, the free energy $\Delta F_{\text {cyl }}$ for a cylindrical segment, spanning the full length of the simulation box in one of the transverse directions, may be obtained from the geometrical formulas: $V=2(\theta-\cos \theta \sin \theta) R^{2} L_{x}$, $A_{s}=4 \theta R L_{x}$, and $A_{f}=A-2 R L_{x} \sin \theta$. The comparison of the free energies for each configuration leads to the geometrical phase diagram presented in Fig. 2, which shows how the MD simulations performed in the smaller box, 256 SDS molecules per monolayer and $A=(9.2 \mathrm{~nm})^{2}$, span through the whole spectrum of shapes, while the larger simulations, 1024 SDS molecules per monolayer and $A=(18.4 \mathrm{~nm})^{2}$, were performed well within the region of stability of the spherical double caps.

\section{COMPUTATION OF CONTACT ANGLES OF NANOSCALE DROPLETS USING THE INTRINSIC SURFACE APPROACH}

Now that we have established the geometrical phase diagram determining the region of stability of the drops in the simulation, we discuss a novel approach to compute the contact angle of the resulting droplets. Recent works have reported computations of contact angles of nanoparticles and nanodroplets at fluid interfaces ${ }^{26}$ as well as droplets adsorbed at solid surfaces. ${ }^{27}$

We show in the following that the computation of contact angles of nanoscale droplets can be efficiently performed using the intrinsic sampling method (ISM) ${ }^{28-31}$ The ISM can be used to construct the local thickness probability distribution $P(h)$ from the analysis of the instantaneous shapes of the 


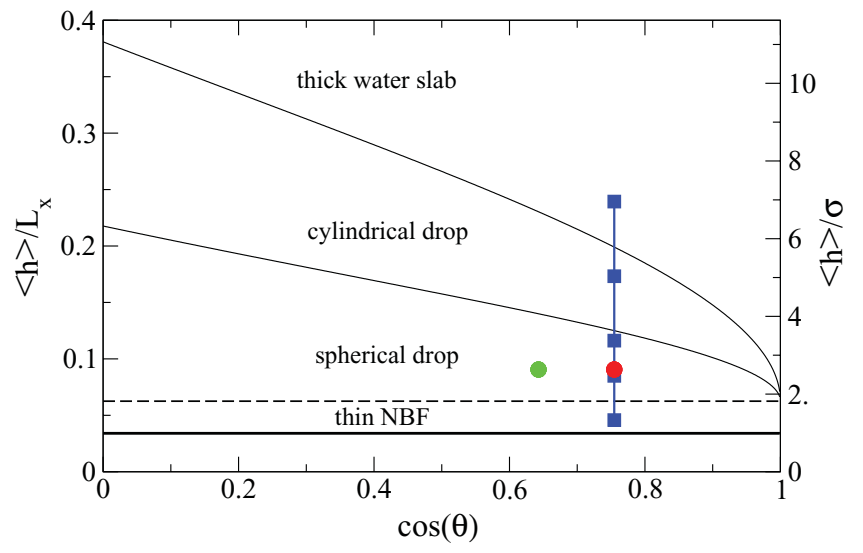

FIG. 2. (Color online) Geometrical diagram for the optimal shape of a water volume within a bilayer as determined by the periodic boundary conditions used in computer simulations over the two transverse directions with length $L_{x}=L_{y}=\sqrt{A}$, and as a function of the contact angle between the thin NBF and the water droplets. The volume of the film $V$, is represented through its mean thickness, $\langle h\rangle=V / A+h_{\mathrm{NBF}}$, scaled with the generic transverse size $L_{x}$ in the left vertical axis. The right vertical axis corresponds to the mean thickness (in units of the molecular diameter of water, $\sigma=0.3166 \mathrm{~nm})$ for the simulations done with box size $L_{x}=$ $9.2 \mathrm{~nm}, T=298 \mathrm{~K}$, and different amounts of water (squares), $n_{w}=N_{w} / N_{\mathrm{SDS}}=11.96,8.6,6.0,4.4$, and 2.0 (from top to bottom) to obtain the surface tension $\gamma\left(N_{w}\right)$. The circles correspond to simulations performed with the larger box, $L_{x}=18.4 \mathrm{~nm}$, which were used to obtain the coexistence of the NBF and the drops with $n_{w}=6.0$, dark (red) circle at $T=298 \mathrm{~K}$ and grey (green) circle at $T=375 \mathrm{~K}$. Notice that in this case, only the left vertical axes is valid. (color online)

SDS monolayers. In our approach, the nominal thickness, $h$, corresponds to the distance between the intrinsic surfaces. The intrinsic surfaces were constructed using the positions of the sulfur atoms in the SDS monolayers (see Ref. 24 for more details). Figure 3 shows $P(h)$ for the systems represented by squares in Fig. 2, which correspond to the smaller system investigated here, 256 SDS. The thickness probability distribution $P(h)$ evolves from a narrow asymmetric peak around the mean thickness of the NBF, to a symmetric Gaussian shape for the thick water slabs, with the mean value set by the amount of water and the width created by the capillary wave fluctuations at the edges of the slab. The intermediate cases, $n_{w}=6$, when the film breaks its planar symmetry to create droplets, give a more interesting probability distribution $P(h)$, which features a double peak, indicating the coexistence between a thin NBF and the water droplet. We note that the formation of a single spherical capped drop, formed by the coalescence of the smaller droplets nucleated in the adhesion process, requires long simulations times $\sim 10^{2}$ ns time scale. Moreover, the relatively small size of the drop results in important shape fluctuations. For this reason, to quantify the shape of the droplet, we performed simulations with larger system sizes. These results are discussed in the following.

Figure 4 shows the thickness distribution at the higher temperature $T=375 \mathrm{~K}$ and $n_{w}=6$, obtained from an average over $12 \mathrm{~ns}$ after allowing $48 \mathrm{~ns}$ for the formation of a lenticular drop with 12288 water and 2048 SDS molecules in a simulation

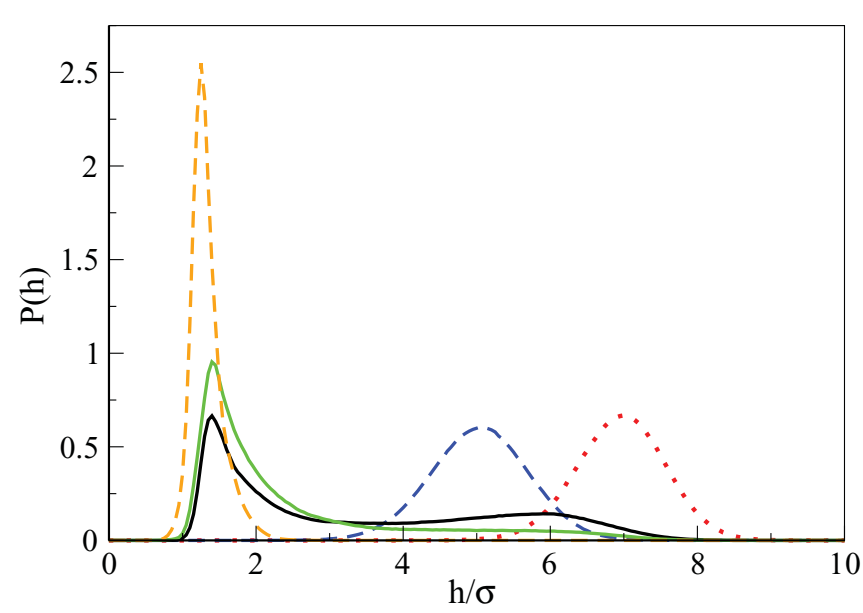

FIG. 3. (Color online) Probability distribution of finding a separation $h$ between the intrinsic surfaces of the two monolayers as a function of the film water content $n_{w}$. The results correspond to the SDS film at $T=298 \mathrm{~K}$ and $L_{x}=9.2 \mathrm{~nm}$. Dotted (red) line: $n_{w}=11.96$, dashed dark grey (blue) line: $n_{w}=8.6$, grey (green) full line: $n_{w}=6.0$, black line: $n_{w}=4.4$, and light dashed (orange) line: $n_{w}=2$. (color online)

box with $L_{x}=L_{y}=18.4 \mathrm{~nm}$. The panel on the left shows the low $h$ part of the distribution $P(h)$, and it is compared with the distribution $P_{\mathrm{NBF}}(h)$ obtained from an average using the thin NBF regions. The typical separation within these regions is about $1.2-1.3$ in units of the molecular diameter of water $(\sigma=0.3116 \mathrm{~nm})$, i.e., only about $0.4 \mathrm{~nm}$. The total thickness of the NBF ( $h \approx 4 \mathrm{~nm}$ ) is obtained adding the length of the SDS tails on the external sides of the film, but for the analysis
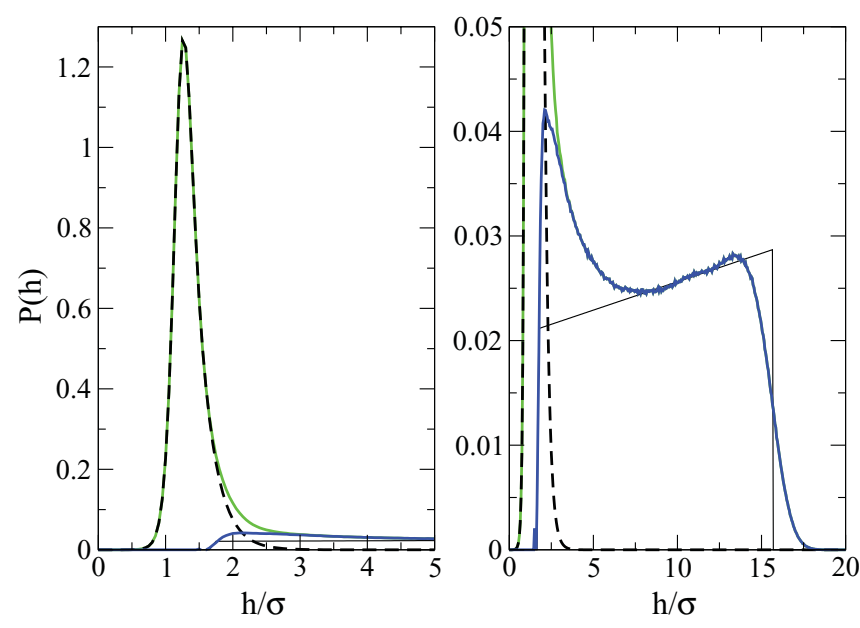

FIG. 4. (Color online) Probability distribution of film thickness $P(h)$ for the SDS film at $T=375 \mathrm{~K}$ with a NBF at coexistence with a lenticular drop, in a system with total transverse size $L_{x}=18.4 \mathrm{~nm}$ and $n_{w}=6$. The grey (green) line shows the simulation results for the total system, and the dashed line the probability distribution over the thin NBF region, normalized to cover a fraction 0.608 of the total transverse area. The dark full line (blue) represents the contribution from the drop $P_{\text {drop }}(h)$, and the thin full line represents the fit to a perfect spherical drop $P_{\mathrm{sph}}(h)$ (see text for details). The left panel presents the detailed view of $P(h)$ for low $h$ (i.e., the NBF) and the right panel shows the large $h$ region (i.e., the water drop). 
of the water drops inside the film it is more convenient to keep the representation in terms of the inner thickness of the SDS bilayer. For $h<1.8 \sigma$, we find that the narrow peak of the total $P(h)$ [grey (green) line] is identical to that in $P_{\mathrm{NBF}}(h)$ (dashed line) rescaled by a factor 0.608 , which represents the fraction of the total area $A$ that is covered by the NBF. Therefore, the distribution of $h$ corresponding to that drop is given by the difference $P_{\text {drop }}(h)=P(h)-0.608 P_{\mathrm{NBF}}(h)$, shown by the grey dark (blue) line in the right panel of Fig. 4 . The integral of $P_{\text {drop }}(h)$ gives the remaining fraction (0.392) of the total area $A$, which represents the projected area of the drop on the $x y$ plane. This drop thickness distribution has to be compared with the histogram of a lenticular drop, with perfect spherical caps: $P_{\mathrm{sph}}(h)=\pi /(2 A)\left[h-h_{0}+2 R \cos (\theta)\right]$ for $h_{o} \leqslant h \leqslant$ $h_{o}+2 R[1-\cos (\theta)]$, where $R$ is the spherical radius and $\theta$ the contact angle at the point where the spherical segments would reach the plane at $h=0$. The parameter $h_{o}$ defines the actual thickness of the film away from the drop, and within this simple representation it should be close the mean value $\langle h\rangle \approx 1.4 \sigma$ in the NBF. $P_{\mathrm{sph}}(h)$ has a trapezoidal shape, with a slope $\pi /(2 A)$ that is independent of the parameters $R, \theta$ and $h_{0}$. Indeed, we observe that the simulation results for $P_{\text {drop }}(h)$ follow precisely that slope over the range $8 \sigma<h<12 \sigma$, and we may therefore use the vertical shift of the straight line to fit $P_{\text {drop }}(h)=P_{\text {sph }}(h)+\pi(h+37.25 \sigma) /(2 A)$ over that range of $h$. Near the top of the drop, for $h>12 \sigma$, the actual shape of $P_{\text {drop }}(h)$ may be regarded as a smoothed version of the sharp edge of $P_{\mathrm{sph}}(h)$. This is expected from the typical fluctuations in the shape of any liquid surface, but since they should not change the projected area of the drop, we may fix the upper threshold of $P_{\mathrm{sph}}(h)$, so that the integral of $P_{\text {sph }}(h)-P_{\text {drop }}(h)$ for $h>8 \sigma$ vanishes. That condition gives $h_{\text {max }}=h_{0}+2 R(1-\cos \theta)=15.68 \sigma$ that together with the above estimates for $P_{\mathrm{sph}}(h)$ and $h_{0}$, give the parameters that define the spherical cap fit to the drop shape, $R=26.45 \sigma$ and contact angle $\theta=41.5^{\circ}$ calculated at the point where $h=h_{0}=1.4 \sigma$.

The estimation of the contact angle involves some uncertainty. Only in the macroscopic limit, $R>>h_{0}$, it would be independent of the estimate of the film thickness. In our case, the estimation of the angle with the same spherical caps extended to the $h=0$ plane would raise the contact angle up to $\theta=44^{\circ}$ as shown in Fig. 5. The deviation between the observed $P_{\text {drop }}(h)$ and the geometrical $P_{\mathrm{sph}}(h)$ shown in the left panel of Fig. 4 is larger at the lower end of the thickness distribution with a strong peak that is approximately twice the predicted value for $P_{\mathrm{sph}}\left(h_{0}\right)$. The interpretation is that the contact between the drop and the NBF is not a mathematical line, but a molecular sized region over which the thickness goes smoothly from the spherical caps to the planar film. The observed distribution $P_{\text {drop }}(h)$ for $2 \sigma<h<7 \sigma$ gives the relevant information on that contact region, and it may be extracted with the hypothesis that $P(h)$ corresponds to an, approximately, axial distribution of thicknesses, i.e., $h(x, y)=h(r)$, with $r^{2}=x^{2}+y^{2}$, and the origin is taken at the drop axis. Within this hypothesis the drop shape is implicitly given by the equation $P[h(r)][d h(r) / d r]=-2 \pi r / A$, where $A$ is the total area over which $P(h)$ is calculated. In the region around the top of the drop $h(0)=h_{\max }$, the equation becomes singular, and it should be integrated assuming that $P(h)$ has
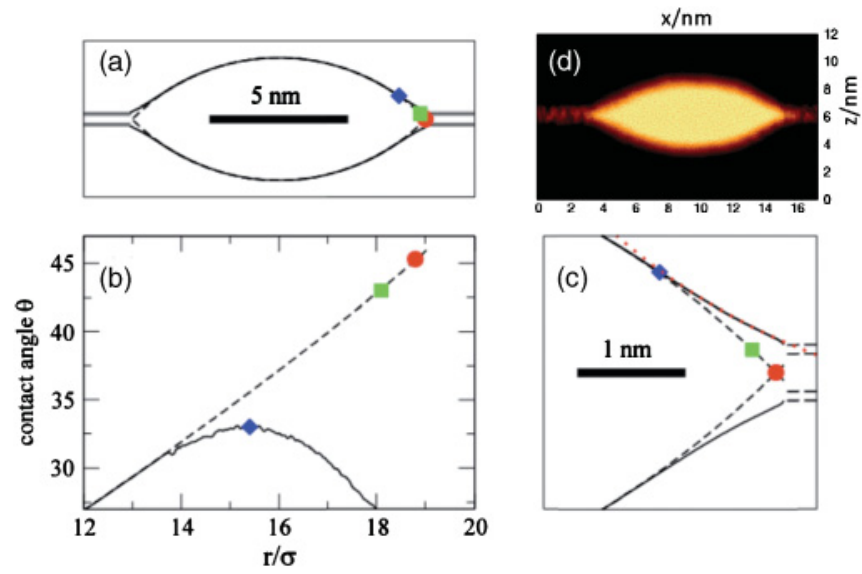

FIG. 5. (Color online) (a)-(c) Drop shape extracted from the thickness probability distributions in Fig. 4 for the SDS film at $T=375 \mathrm{~K}$. The dashed lines correspond to the spherical cap representation, while the full lines are obtained assuming perfect axial symmetry. (a) A general view of the full water drop, while panel (c) focus on the contact region, with the dotted (red) line representing a circular fit to the shape of the meniscus. The parallel horizontal lines give the estimates for the NBF. Panel (b) gives the local angle of the surface with respect to the horizontal NBF, both along the sphere cap fit (full line) and the meniscus shape extracted from $P(h)$ (dashed line). The symbols represent the different choices to evaluate the contact angle: circle and square for the $h=0$ and $h=h_{0}$ intersections of the spherical caps respectively. The diamond is the maximum local value of the angle, before the flattening of the meniscus shape. (d) Contour plot of the density profile of the water droplet obtained directly from the analysis of the simulations at 375 $\mathrm{K}$. The results correspond to an average over the last $12 \mathrm{~ns}$ of the simulation.

the perfect spherical shape of $P_{\mathrm{sph}}(h)$. Because in the contact region, the presence of irregular fluctuations does not have a significant effect the mean shape of the drop may be calculated from

$$
r=\left[\frac{A}{\pi} \int_{h(r)}^{h_{\max }} d h P(h)\right]^{1 / 2} .
$$

The results in Fig. 5 show that the contact region spans about $3 \sigma$ around the mathematical contact line, and the local thickness deviates up to one molecular diameter $\sigma$ from the spherical cap prediction. In the contact region, the curvature of the line $h(r)$ changes its sign, with respect to the curvature in the spherical cap. The dotted line of the panel (c) of Fig. 5 shows that the shape of the meniscus formed by $h(r)$ in the contact region is very well fitted by a circumference of radius $R_{\text {men }}=18.8 \sigma$ with the opposite curvature of the axial radius $R \sin (\theta)=18 \sigma$, so that the meniscus has nearly null mean curvature. The local angle of the drop $\theta(r)=\arctan \{[d h(r)] / d r\}$ is shown in Fig. 5(b). As $r$ increases from the drop axis toward the contact region, $\theta(r)$ reaches a maximum at $\theta_{\max }=34^{\circ}$. We find that nowhere along the actual surface of the drop the local contact angle reaches the value $\theta_{\mathrm{sph}}\left(h_{0}\right)=41.5^{\circ}$ extracted from the spherical cap fit to the upper half of $P_{\text {drop }}(h)$, and which should be the relevant value to be used in Youngs equation. Of course, these different routes to estimate the contact angle should agree for large 

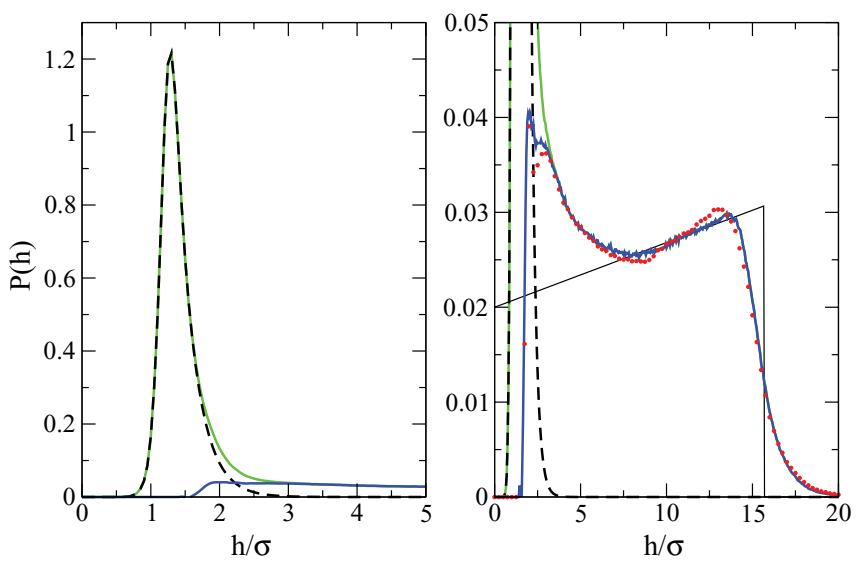

FIG. 6. (Color online) Probability distribution of film thickness $P(h)$ in the SDS Film. The lines have the same meaning as in Fig. 4 but for $T=298 \mathrm{~K}$. The dark grey (blue) and dotted lines (red) in the right panel, represent the contribution of the droplet to the probability distribution sampled over two different 12 ns intervals. The dashed line represents the probability distribution for the thin NBF region, normalized to cover a fraction 0.597 of the total transverse area. The left panel presents the detailed view of $P(h)$ for low $h$ (i.e., the NBF) and the right panel shows the large $h$ region (i.e., the water drop).

drops, when $R$ is much larger than the contact region. In our simulations, with a drop formed by $\sim 10^{4}$ water molecules, the finite size effects are still important, and produce significant differences in the estimation of $\theta$.

The same analysis has been carried out for the drop formed at lower temperature, $T=298 \mathrm{~K}$, giving an estimate of $R=22.5 \sigma$ for the radius and $\theta=50^{\circ} \pm 2^{\circ}$ for the contact angle. We note that the increase in contact angle observed at lower temperatures agrees with the predictions of WT theory. However, the low temperature results show some important differences with those at $T=375 \mathrm{~K}$, reflecting some of the possible peculiarities of black films as wetting systems. First of all, the dynamics for the formation of the drop is much slower at this temperature, and long simulation spanning more about $0.2 \mu s$ were needed to form a single drop with good axial symmetry. Even after such long relaxation time, the sampling of $h$ over 12 ns intervals still gave important differences in the shape of $P_{\text {drop }}(h)$, particularly in the linear region used in the fit to $P_{\mathrm{sph}}(h)$, as shown in Fig. 6. Also, the direct comparison of the fitted spherical caps with snapshots of the system in Fig. 7 shows a poorer agreement than at high temperature.

The direct observation of the SDS molecules in snapshots of the simulation gives the clue for the behavior observed at low temperature. First of all, the mobility of the surfactant molecules in the NBF is strongly reduced with respect to what is observed at high $T$, with a concomitant slowing down in the diffusion of water in the thin film, and hence the coalescence of the nucleated droplets into a single drop. A second effect is connected to the variation of the concentration of surfactants in the region near the apex of the drop, which is significantly reduced. The drop features regions that are completely depleted from surfactants. Hence in that region, the surface tension will be larger than in regions where the surfactant concentrates. Our results point towards a surface phase transition in the surfactant monolayers, with a two-
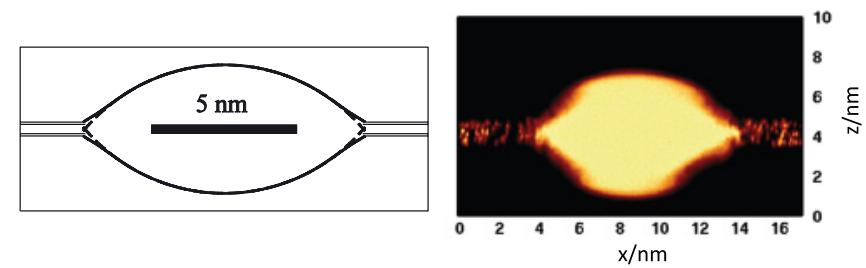

FIG. 7. (Color online) (Left) Drop shape extracted from $P(h)$ for the SDS system at $T=298 \mathrm{~K}$. The broken line is the spherical cap fit and the continuous thick line is the result of assuming that $P(h)$ has perfect axial symmetry. (Right) Contour plot of the density profile of the water droplet obtained directly from the analysis of the simulations at $298 \mathrm{~K}$. The results correspond to an average over the last $12 \mathrm{~ns}$ of a simulation spanning $200 \mathrm{~ns}$.

dimensional condensation. Therefore regarded as the substrate of a wetting system, the SDS bilayer reacts to the formation of the wetting phase. i.e., the droplets, in a complex way. Because the drops form inside the film, the substrate, i.e. the surfactant monolayers, have to change their total area. As the monolayers have the freedom to redistribute their local density, the amount of surfactant per unit area at the droplet apex might be different from that of the NBF. In a typical experimental situation in which the film is in contact with a surfactant reservoir, the amount of surfactant in the coexisting NBF and the drop surface would be fixed by the chemical potential of the surfactant. However, in a canonical ensemble simulation or in an experiment with an isolated film in a fixed area frame, the distribution of the surfactant between the two coexisting structures may result in a more complex situation, with a substrate phase transition, which is atypical in the usual wetting systems.

We note that the simulations of the smaller system, with $L_{x}=9.2 \mathrm{~nm}$ and $256 \mathrm{SDS}$ molecules per layer, could only produce smaller droplets, for which the contact region spanned too much of the total drop shape and the fluctuations where more important. Therefore a good quantitative analysis for the drop shape becomes impossible, although the observed shapes for the thickness distribution $P(h)$ could still be semiquantitatively interpreted using the approach discussed above for the larger drops.

\section{WETTING TRANSITION THEORY AND NEWTON BLACK FILM FORMATION}

The formation of droplets inside the films supports the existence of a wetting transition in Newton black films. The WT theory provides a general approach to predict the wetting behavior from the film's surface tension, $\gamma(\Gamma, T, \mu)$, as a function of the adsorption $\Gamma$, i.e., the excess number of water molecules per unit area, the temperature $T$, and the chemical potential. At bulk coexistence, $\mu_{\text {sat }}$, and $\mathrm{T}$ below the wetting temperature $T_{w}$, the thermodynamic equilibrium state (TES) corresponds to a thin water layer and it is given by the minimum of $\gamma\left(\Gamma ; T, \mu_{\text {sat }}\right)$. The layer becomes macroscopically thick for $T>T_{w}$. The transition between thin and thick layers may be continuous (second order) or discontinuous (first order), the latter involving an energy barrier $\Delta \gamma$ between the thin film and the macroscopic layer state. The surface field and surface enhancement, or the 


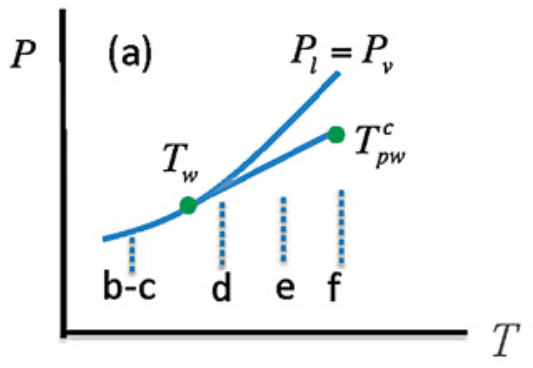

$\mathrm{T}<\mathrm{T}_{\mathrm{w}}$ NBF stable for $\Pi=0$

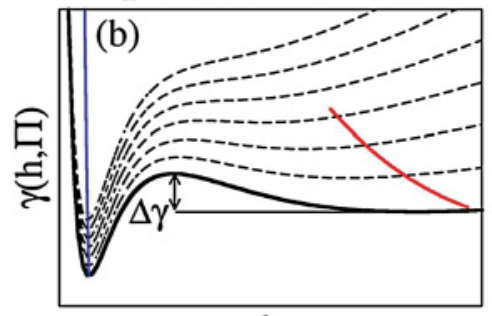

$\mathrm{h}$

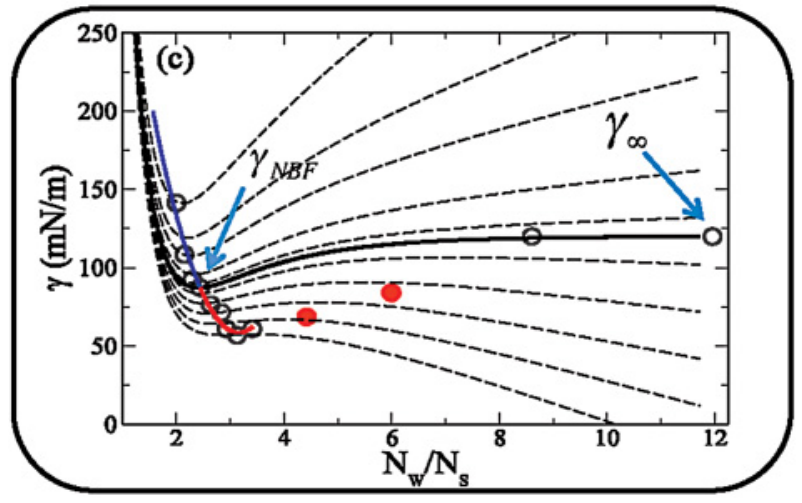

T $>$ Tw NBF metastable for $\Pi=0$

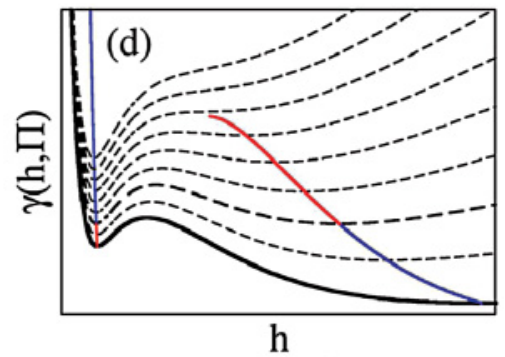

T $>$ Tw NBF unstable for $\Pi=0$

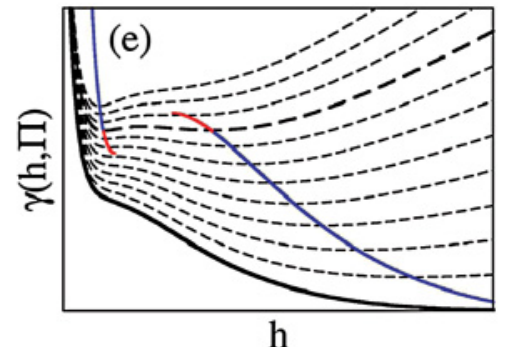

$T \geq T_{p w}^{c}$

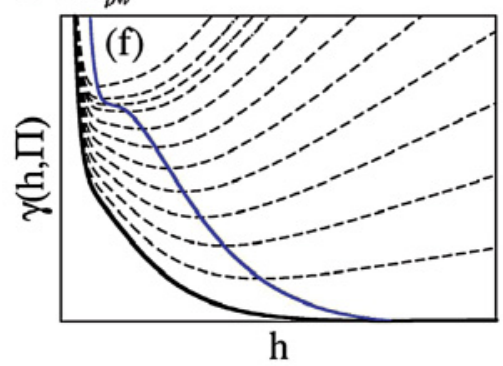

FIG. 8. (Color online) (a) Sketch of a generic pressure-temperature phase diagram showing the wetting $T_{w}$ and critical prewetting $T_{p w}^{c}$ temperatures. The saturation line, $P_{l}=P_{v},(\Pi=0)$ is also shown. (b) Sketch of the surface tension $\gamma(h ; \Pi)$ versus the film thickness $h$ in a partial wetting situation with $\Delta \gamma>0$, which would lead to the complete wetting, whereas the sketches (d)-(f) span the prewetting line. The full line in these panels indicates the saturation condition, $\Pi=0$, and the dashed lines correspond to $\Pi>0$, where $\gamma(h ; \Pi)=\gamma(h ; 0)+\Pi h$ (see Sec. III and the Appendix for a discussion on the construction on these diagrams). The location of the thermodynamically stable (dark grey or blue) and metastable phases (light grey or red) have been indicated. The thick dashed lines indicate prewetting coexistence between NBF and CBF. The circles in panel (c) show our molecular dynamics computer simulation results of the surface tension of sodium dodecyl sulfate black films as a function of the number of water molecules per surfactant at a partial wetting situation. Note that unlike in sketch (b), the conjectured dashed lines are also shown for $\Pi<0$. The full circles represent systems with inhomogeneous thicknesses, where NBF regions coexist with small water droplets. The open circles represent stable and metastable states. The arrows indicate the surface tensions of the equilibrium NBF and the corresponding macroscopic thick film.

strength and range of the substrate-adsorbate interactions determine $\mathrm{T}_{w}$ and $\Delta \gamma \cdot{ }^{32}$ When the wetting phase is away from bulk coexistence, the surface tension increases with $\Gamma$ as $\gamma(\Gamma ; T, \mu)=\gamma\left(\Gamma ; T, \mu_{\text {sat }}\right)+\Delta \mu \Gamma$, shifting the relative stability of the thin and thick layers for $T>T_{w}$. Any first order WT features a prewetting line, i.e., a first-order surface phase transition between thin and thick films at $\Delta \mu(T)>0$, over a range of temperatures from $T_{w}$ to the critical prewetting temperature $T_{p w}^{c}$ [see Fig. 8(a)].

The accessible variable in experiments of black films is the film thickness $h$, which plays the role of the adsorption, while the disjoining pressure controls $\Delta \mu$. The experimental disjoining pressure curves ${ }^{7,11}$ closely follow the predictions of the WT theory. At saturated conditions, $\Pi=0$, and $T<T_{w}$ [see Fig. 8(b)] the film surface tension reaches a minimum that corresponds to the formation of the NBF, first discussed by Newton, ${ }^{1}$ and recently investigated using experiments with ionic surfactants. ${ }^{13,14}$ This is a partial wetting state. At $\Pi=0$ one can induce complete wetting at $T>T_{w}$, by increasing the temperature or changing the amphiphile concentration, then the NBF becomes metastable [see Fig. 8(d)] or unstable [see Figs. 8(e) and 8(f)] with respect to the thicker CBF. In the partial wet state, a contact angle between the NBF and the surface of the inner water droplets must appear, a notion that is compatible with early experimental studies. ${ }^{17}$ Under positive $\Pi$, the stable NBF would become slightly thinner, but without qualitative changes in its structure, again compatible with the apparent insensitivity of the NBF thickness to pressure changes, reported in several experiments. ${ }^{7,11}$ A high-energy barrier in $\gamma(h ; 0)$ may inhibit the formation of the NBF from a 
thick film, and a moderate increase in $\Pi$ may keep the system in a metastable CBF [light grey (red) line in Fig. 8(b)]. The value of $\Pi$ needed to induce the spontaneous formation of the NBF may be reduced or even eliminated by lowering the barrier $\Delta \gamma$, e.g., by increasing the amount of salt.

For $T>T_{w}$, and $\Delta \gamma>0$, the theory predicts that the $\mathrm{NBF}$, i.e., the partial wetting state, moves away from bulk coexistence, along the prewetting transition line, $\Pi_{p w}(T)>0$, where it coexists with the thicker $C B F$. This is the CBF-NBF transition reported in experiments of non-ionic surfactants, see Fig. 2 of Ref. 11, which corresponds to the sequence (d)-(e)-(f) in our Fig. 8. For $\Pi<\Pi_{p w}(T)$, the thin NBF becomes metastable with respect to the thicker CBF. The shallow minimum of $\gamma(\Gamma ; T, \mu)$ makes the film very sensitive to changes in $\Pi$ and/or $\Delta \gamma$, i.e., disjoining pressure and/or salinity. The range of metastability for NBFs and CBFs at each side of $\Pi_{p w}(T)$, and the difference in thickness between the CBF and NBF become smaller as T increases toward the critical prewetting point [see Figs. 8(d) and 8(e)]. For $T>T_{p w}^{c}$, a continuous change in the film thickness will be observed [see Fig. 8(f)]. Recently, the CBF-NBF transition has been discussed in the context of liquid-vapor phase transitions. ${ }^{33}$ This idea is reminiscent of the interpretation of the wetting transitions in terms of bulk phase transitions, a notion that was introduced shortly after the discovery of the WT theory. ${ }^{32}$

The dependence of $\gamma(h ; \Pi)$ with $h$ (see Fig. 8) agrees with the DLVO theory, ${ }^{5,6}$ which considers a balance of electrostatic repulsion between the two layers and their effective attraction due to van der Waal forces. That theory explains the influence of salt concentration on the barrier height $\Delta \gamma$. Depending on this height a first order WT, with a long (prewetting) NBF-CBF coexistence line and large hysteresis, may change to a weakly first order, or even a continuous wetting transition.

Figure 8(c) shows our surface tension data (see Fig. 7) represented in the context of the WT theory. These data were used to find the saturation conditions of water, i.e., $\Delta \mu=0$ (see Sec. III). An adhesion transition is observed with six water molecules per SDS. This state lies between the line of under/supersaturated NBF, extended at both sides of $\Delta \mu=0$, which corresponds to 1-2 water molecules per SDS, and the plateau defined by $\gamma_{\infty}=0.120 \mathrm{~N} / \mathrm{m}$, which defines a state where thick water slabs with $\mu\left(N_{w}\right)=\mu_{\text {sat }}$ form.

The simulation of large system sizes has enabled us to show that in the adhesive state the excess water in the NBF is expelled from the film, forming a lenticular drop. This is the behavior expected in a partial-wetting system, $T<T_{w}$, with the thin NBF coexisting with a large inclusion of the wetting phase. The contact angle of the water droplets, obtained from the analysis of the simulations, $\theta=50^{\circ} \pm 2^{\circ}(298 \mathrm{~K})$ agrees with Youngs equation prediction, $\gamma_{\infty} \cos \theta=\gamma_{\mathrm{NBF}}$, using the surface tensions indicated in Fig. 8(c) for $\Delta \mu=0$. Increasing the temperature, $T=375 \mathrm{~K}$, the contact angle decreases to $\theta=41.5^{\circ} \pm 2^{\circ}$. This is again the expected behavior for a system approaching complete wetting.

\section{PECULIARITIES OF WETTING TRANSITIONS IN NBFS}

The bilayer formed by the SDS surfactant molecules acts as the "substrate" in a typical partial wetting problem, but contrary to the case of a liquid drop on a plane solid surface, the SDS film is not "inert," and it may react to the formation of the water drop. This situation arises naturally in our canonical MD simulations, which are performed at fixed number of SDS molecules $N_{w}$ and fixed cross-sectional area, $A$. As discussed above this can result in a surface surfactant concentration that may by higher in the NBF, and lower at the apex of the drop surface, so that the mean surfactant concentration $N_{w} / A$ is constant. Moreover, the total surface to be covered by the SDS films changes during the formation of the drop, and it depends on the shape of the drop. Therefore, although Young's equation, $\cos (\theta)=\gamma_{\mathrm{NBF}} / \gamma_{\infty}$ is still valid, we have to be aware that the values of $\gamma_{\mathrm{NBF}}$ and $\gamma_{\infty}$ employed above, cannot be obtained directly from those of uniform thin films and thick slabs with the same fixed surfactant concentration $N_{w} / A$. As a matter of fact, the analysis of the simulations with the larger number of surfactants, $T=298 \mathrm{~K}$ and $n_{w}=N_{w} / N_{\text {SDS }}=6$, which corresponds to the adhesive films, indicates that the area per surfactant in the self assembled NBF is $31.4 \AA^{2}$ per surfactant, instead of the average $A / N_{\mathrm{SDS}}=33 \AA^{2}$. Another canonical MD simulation of a thin film with that surfactant surface concentration gives a surface tension of $\gamma_{\mathrm{NBF}} \approx 77 \mathrm{mN} / \mathrm{m}$. Consistently, we should assume that the higher concentration of SDS on the NBF region implies a lower concentration on the surface of the liquid drop, so that the value of $\gamma_{\infty}$ in this case would be larger than $120 \mathrm{mN} / \mathrm{m}$ estimated from the thick water slabs with $A / N_{\text {SDS }}=33 \AA^{2}$.

Overall, the accurate prediction of the contact angle would require information on the dependence of the surface tension with respect to the surfactant concentration, both in the thin NBF and in the single SDS monolayer (or the thick water slab). The true thermodynamic equilibrium state for the liquid drop inside the BF requires the equality of the surfactant chemical potential in the two regions. This would produce small changes in the water/surfactant ratio, in the surface tensions and in the contact angle, with respect to those estimated for fixed surfactant concentrations. However, this complication is only a minor trouble, and it does not have a major impact in the prediction of the NBF structure from canonical ensemble computer simulations.

The main conclusion of our analysis is that NBF may indeed be interpreted as equilibrium structures. They can be identified in canonical MD simulations as structures in coexistence with water drops, i.e., a situation that is qualitatively similar to the partial wetting of a drop on a substrate.

\section{CONCLUSIONS AND FINAL REMARKS}

The connection between black films and the wetting transition provides a notion to study these films using the powerful theoretical techniques that have been developed to understand fluctuations and dynamics in wetting systems, such as liquids on a solid substrate, liquid-liquid interfaces, surface segregation of solid alloys, surface ferromagnetism, or liquid crystal interfaces. Black films can now be added to this list, with the peculiarity that the wetting phase is inside the bilayer substrate, so that it may play a more active role than in the usual wetting of inert substrates. The relevant variables, $\Delta \gamma$, $\Delta \mu=\mu_{\text {sat }}-\mu$, and $\mathrm{T}_{w}$, which control the wetting behavior of a system, may be controlled in BFs through the salinity of 
water, the disjoining pressure, and the chemical composition of the amphiphile. BFs thus provide unique systems to readily study the prewetting transition and the spreading behavior in a system with frustrated complete wetting ${ }^{20}$, since the wetting phase (water) has a higher refractive index than the (vapor) bulk phases on both sides of the film, a situation very difficult to find in the usual wetting systems.

The possibility that the NBF substrate, i.e., the surfactant layers are not inert, i.e., that their surface concentration can change depending on the experimental conditions, provides a variable to create and control nanostructured films.

We have to point out that the generic WT theory does not explain the stability of the substrate, i.e., the surfactant layers. The (meta)stability of the thin NBF with respect to its rupture, which depends on the molecular structure of the hydrated amphiphiles, may only be understood with molecular models that include the relevant characteristics of these molecules. In this respect, our extensive MD simulations show that the adhesive membrane is very stable, and arises naturally when the polar heads of the SDS molecules share a small amount of water.

Our work rationalizes previous experimental studies and we anticipate it may provide a framework to design thin films with uses as two-dimensional templates to assist the assembly of complex nanoscale structures ${ }^{34,35}$ as well as to understand the physical mechanism of membrane adhesion in biological systems. ${ }^{36}$

\section{ACKNOWLEDGMENTS}

We would like to acknowledge the Imperial College High Performance Computing Service for providing computational resources. Financial support for this work was provided by The Royal Society project "Intrinsic Structure of Aqueous Interfaces" and the Dirección General de Investigación, Ministerio de Ciencia y Tecnología of Spain, under Grant No. FIS2010-22047-C05, and by the Comunidad Autónoma de Madrid under the R\&D Program of activities MODELICOCM/S2009ESP-1691. H.M. would like to thank the Universidad Autonoma de Madrid for the award of a FPU-UAM doctoral grant. F.B. would like to thank the EPSRC for the award of a Leadership Fellowship (EP/J003859/1).

\section{APPENDIX: CALCULATION OF THE SURFACE TENSION CURVES}

The surface tension $\gamma(h ; \Pi)$ curves showed in the sketches of Fig. 8 have been obtained using the model proposed in Ref. 11. We assume that $\gamma(h ; \Pi=0)$ contains a steric repulsion term $B / h^{8}$ between the surfactant molecules, an attractive van der Waals interaction $A / h^{2}$, and the screened electrostatic repulsion given by the linearized form of the Poisson-Boltzmann equation:

$$
\gamma(h, \Pi=0)=\frac{B}{h^{8}}-\frac{A}{h^{2}}+C \exp (-\kappa h),
$$

where $C$ depends on the surface charge, and the Debye screening $\kappa^{-1}$ of the salt concentration. Note that we consider $\gamma(h ; \Pi=0)$, while in Ref. 11 , the authors considered the disjoining pressure: $\Pi(h)=-d \gamma(h) / d h$. Our values for the constants $B, A, C$, and $\kappa$ have been chosen in order to reproduce the correct physical behavior, with sharp maxima and minima in the surface tension curves. We note that we have not attempted to model a specific experimental system. In all the sketches shown in Fig. 8, the dimensionless values $A=1 / 3, B=4 / 3, \kappa=1$ were used. The sketches (d)-(f) were obtained by changing the barrier height. We considered the values $C=2.5,3,4$, and 5 , respectively. The curves with disjoining pressure $\Pi=0$ were obtained by adding a linear term to the $\Pi=0$ function,

$$
\gamma(h ; \Pi)=\gamma(h ; \Pi=0)+\Pi h,
$$

choosing values for the disjoining pressure that qualitatively cover the relevant range. Therefore our sketched form for $\gamma(h ; \Pi)$ were done using the same theoretical framework considered in Ref. 11, but again we insist with have not attempted here to give a realistic quantitative description of specific experimental systems, but to reflect the generic wetting behavior expected in experimental situations.

The sketches of $\gamma\left(n_{w} ; \mu\right)$ presented in Fig. 8(c) were constructed to quantitatively fit the results of the MD simulations for the equilibrium values of $\gamma\left(n_{w} ; \mu\right)$. Since these data do not give information on the possible metastability barrier, we have used the simplest possible description for a partial wetting situation, namely, we have used two exponential functions:

$$
\gamma\left(n_{w} ; 0\right)=\gamma_{\text {thick }}+A \exp \left(-\alpha n_{w}\right)+B \exp \left(-\beta n_{w}\right),
$$

where $n_{w}=N_{w} / N_{\mathrm{SDS}}$.

The thick layer value $\gamma_{\text {thick }}=0.12 \mathrm{~N} / \mathrm{m}$ is directly obtained from the results with $n_{w}>6$. The parameters $A=5.9 \mathrm{~N} / \mathrm{m}$, $B=0.173 \mathrm{~N} / \mathrm{m}, \alpha=2.7$, and $\beta=0.59$ are fitted to get the observed values for the position and the minimum of

$$
\gamma\left(n_{w} ; \Delta \mu\right)=\gamma\left(n_{w} ; 0\right)+\frac{\Delta \mu N_{w}}{A_{0}},
$$

when water is shifted away from bulk coexistence conditions. Notice that the absence of a metastability barrier, i.e., $\Delta \gamma=$ 0 , in this expression, the surface tension would produce continuous, rather than first order wetting transitions, for $T>T_{W}$. However, that distinction is irrelevant for $T<T_{W}$, as the only stable state is the thin NBF, and the structure of the minimum of $\gamma\left(n_{w} ; \Delta \mu\right)$ cannot be used to infer the shape of that function in the unstable region.

\footnotetext{
*pedro.tarazona@uam.es

†hector.martinez@uam.es

‡e.chacon@icmm.csic.es

§f.bresme@imperial.ac.uk
}

${ }^{1}$ I. Newton, Opticks: or a Treatise of the Reflections, Refractions, Inflections and Colours of Light, Book 2,4th ed. (William Innys, New York, 1730), Part I, Obf. 17.

${ }^{2}$ V. Bergeron, J. Phys. Condens. Matter 11, R215 (1999). 
${ }^{3}$ V. Bergeron, A. Waltermo, and P. Claesson, Langmuir 12, 1336 (1996).

${ }^{4}$ N. Cuvillier, F. Millet, V. Petkova, M. Nedyakov, and J.-J. Benattar, Langmuir 16, 5029 (2000).

${ }^{5}$ M. Jones, K. Mysels, and P. Sholten, Trans. Faraday Soc. 62, 1336 (1966).

${ }^{6}$ D. Sentenac, A. Schalchli, M. Nedyalkov, and J. Benattar, Faraday Discuss.104, 345 (1996).

${ }^{7}$ D. Exerowa, T. Kolarov, and K. Khristov, Colloids Surf. 22, 171 (1987).

${ }^{8}$ J. Faraudo and F. Bresme, Phys. Rev. Lett. 92, 236102 (2004).

${ }^{9}$ D. Langevin, Adv. Colloid Interface Sci. 88, 209 (2000).

${ }^{10}$ K. Mysels, J. Phys. Chem. 68, 3441 (1964).

${ }^{11}$ V. Casteletto, I. Cantat, D. Sarker, R. Bausch, D. Bonn, and J. Meunier, Phys. Rev. Lett. 90, 048302 (2003).

${ }^{12}$ J. Bibette, T. Mason, H. Gang, D. Weitz, and P. Poulin, Langmuir 9, 3352 (1993).

${ }^{13}$ O. Bélorgey and J. J. Benattar, Phys. Rev. Lett. 66, 313 (1991).

${ }^{14}$ P. Poulin, F. Nallet, B. Cabane, and J. Bibette, Phys. Rev. Lett. 77, 3248 (1996).

${ }^{15}$ M. Aronson and H. Princen, Nature (London) 286, 370 (1980).

${ }^{16} \mathrm{~S}$. Bailey, S. Chiruvolu, J. Israelachvili, and J. Zasadzinski, Langmuir 6, 1326 (1990).

${ }^{17}$ K. Mysels, H. Huisman, and R. Razouk, J. Phys. Chem. 70, 1339 (1966).

${ }^{18}$ J. Cahn, J. Chem. Phys. 66, 3667 (1977).

${ }^{19}$ H. Nakanishi and M. E. Fisher, Phys. Rev. Lett. 49, 1565 (1982).
${ }^{20}$ D. Bonn, J. Eggers, J. Indekeu, J. Meunier, and E. Rolley, Rev. Mod. Phys. 81, 739 (2009).

${ }^{21}$ B. Hess, J. Chem. Thoery Comput. 4, 435 (2008).

${ }^{22}$ J. Abascal and C. Vega, J. Chem. Phys. 123, 234505 (2005).

${ }^{23}$ F. Bresme and J. Faraudo, Langmuir 12, 5127 (2004).

${ }^{24}$ F. Bresme, E. Chacón, H. Martínez, and P. Tarazona, J. Chem. Phys. 134, 214701 (2011).

${ }^{25}$ T. Darden, D. York, and L. Pedersen, J. Chem. Phys. 98, 10089 (1993).

${ }^{26}$ F.Bresme and M. Oettel, J. Phys. Condens. Matter 19, 413101 (2007).

${ }^{27}$ F. Ould-Kaddour and D. Levesque, J. Chem. Phys. 135, 224705 (2011).

${ }^{28}$ E. Chacón and P. Tarazona, Phys. Rev. Lett. 91, 166103 (2003).

${ }^{29}$ P. Tarazona and E. Chacón, Phys. Rev. B 70, 235407 (2004).

${ }^{30}$ F. Bresme, E. Chacón, and P. Tarazona, PhysChemChemPhys. 10, 4704 (2008).

${ }^{31}$ E. Chacón, E. M. Fernández, D. Duque, R. Delgado-Buscalioni, and P. Tarazona, Phys. Rev. B 80, 195403 (2009).

${ }^{32}$ D. Sullivan and M. T. da Gama, Wetting Transition and Multilayer Adsorption at Fluid Interfaces, in Fluid and Interfacial Phenomena, edited by C.A. Croxton (Wiley, New York, 1986).

${ }^{33}$ C. Stubenrauch and R. Strey, Langmuir 20, 5185 (2004).

${ }^{34} \mathrm{G}$. Andreatta, Y. Wang, F. Lee, A. Polidori, P. Tong, B. Pucci, and J. Benattar, Langmuir 24, 6072 (2008).

${ }^{35}$ J. Jin, W. Bu, and I. Ichinose, Langmuir 26, 10506 (2010).

${ }^{36}$ E. Sackmann and R. Bruinsma, Chem. Phys. Chem. 3, 262 (2002). 